\title{
Colloidal dual-band gap cell for photocatalytic hydrogen generation
}

Wei Li, ${ }^{a, b, \dagger}$ Graeme O`Dowd, ${ }^{a}$ Thomas J. Whittles, ${ }^{\text {a }}$ David Hesp, ${ }^{\text {a }}$ Yvonne Gründer, ${ }^{\text {a }}$ Vinod R. Dhanak, ${ }^{\text {a }}$ Frank Jäckel ${ }^{\mathrm{a}, \dagger}$

We report that the internal quantum efficiency for hydrogen generation in spherical, Pt-decorated CdS nanocrystals can be tuned by quantum confinement, resulting in higher efficiencies for smaller than for larger nanocrystals ( $17.3 \%$ for $2.8 \mathrm{~nm}$ and $11.4 \%$ for $4.6 \mathrm{~nm}$ diameter nanocrystals). We attribute this to a larger driving force for electron and hole transfer in the smaller nanocrystals. The more efficient energy conversion in smaller nanocrystals enables a novel colloidal dual-band gap cell utilising differently sized nanocrystals and showing larger external quantum efficiencies than cells with only one size of nanocrystals ( $9.4 \%$ for $2.8 \mathrm{~nm}$ particles only and $14.7 \%$ for $2.8 \mathrm{~nm}$ and $4.6 \mathrm{~nm}$ nanocrystals). This represents a proof-of-principle for future colloidal tandem cell.

\section{Introduction}

Hydrogen, produced by splitting water using sun-light, is currently considered as one component of a future sustainable energy supply. ${ }^{1}$ Colloidal, co-catalyst decorated semiconductor nanocrystals have recently received considerable attention for photocatalytic hydrogen generation due to their widely tuneable optical and electronic properties..$^{2-5}$ To generate hydrogen photocatalytically, the colloidal semiconductor nanocrystal (SNC) absorbs light, generating an electron hole-pair. The photo-generated charges are then separated, as illustrated in the inset of Fig. 1a, with the electron being transferred to the co-catalyst where it reduces water to generate hydrogen, while the hole is removed by a sacrificial hole scavenger. Recent studies have established hole removal and thus suppression of electron hole recombination as the major rate limiting step for efficient hydrogen generation. ${ }^{6}$ Efficient hole removal has been demonstrated by adjusting the hole scavenger redox potential or introducing a redox-shuttle. ${ }^{7,8}$ A key parameter in the electron transfer and hole scavenging rate is the energetic off-set between the electronic states of the semiconductor with respect to the co-catalyst and the hole scavenger's redox-potential, respectively, as it defines the driving force $\Delta \mathrm{G}$ for the transfer/scavenging reaction. ${ }^{9}$ In SNCs these energetic off-sets can in principle be tuned by controlling the SNC-size and, via quantum confinement, the optical and electronic properties. ${ }^{10}$ Recent studies investigated the size-dependence of electron transfer kinetics to co-catalysts. ${ }^{11,12}$ However, this strategy has not yet been used to optimise overall hydrogen generation in co-catalyst decorated colloidal nanocrystals. Here we show that size-controlled CdS nanospheres decorated with Pt-clusters exhibit internal quantum efficiencies for photocatalytically hydrogen generation which monotonously increase with decreasing particle size from $11.4 \%$ (4.6 nm nanocrystals) to $17.3 \%$ ( $2.8 \mathrm{~nm}$ nanocrystals). We use this size-dependence to realise a colloidal dual-band gap cell in which external quantum efficiencies increase from $9.4 \%$ ( $2.8 \mathrm{~nm}$ particles only) to $14.7 \%$ ( $2.8 \mathrm{~nm}$ and $4.6 \mathrm{~nm}$ particles) under visible light illumination ( $350-480 \mathrm{~nm}$ ). Our results demonstrate that the improved efficiencies in the dual-band gap cell originate from the smaller

\footnotetext{
a. Department of Physics and Stephenson Institute for Renewable Energy, The

University of Liverpool, Chadwick Building, Peach Street, Liverpool, L69 7ZF, UK

b. Present address: Chemical Engineering \& Applied Chemistry and European

Bioenergy Research Institute, Aston University, Aston Triangle, Birmingham B4 TET, UK

+w.li8@aston.ac.uk and fjaeckel@liverpool.ac.uk

Electronic Supplementary Information (ESI) available: full experimental details and additional experimental results. See DOI: 10.1039/x0xx00000x
} 
nanocrystals converting photons more efficiently into hydrogen by providing larger driving forces $\Delta \mathrm{G}$ for the charge transfer reaction, while larger nanocrystals use lower energy photons not absorbed by the smaller nanocrystals. This prototype is a proof of principle that is applicable to other solar fuels such as methane and methanol derived from $\mathrm{CO}_{2}$ reduction as well as other semiconductor-cocatalyst systems. ${ }^{13}$ The dual-band gap cell represents a proof-of-principle for a colloidal tandem cell in which the two compartments would be electrically connected through a membrane sharing the same electrolyte.
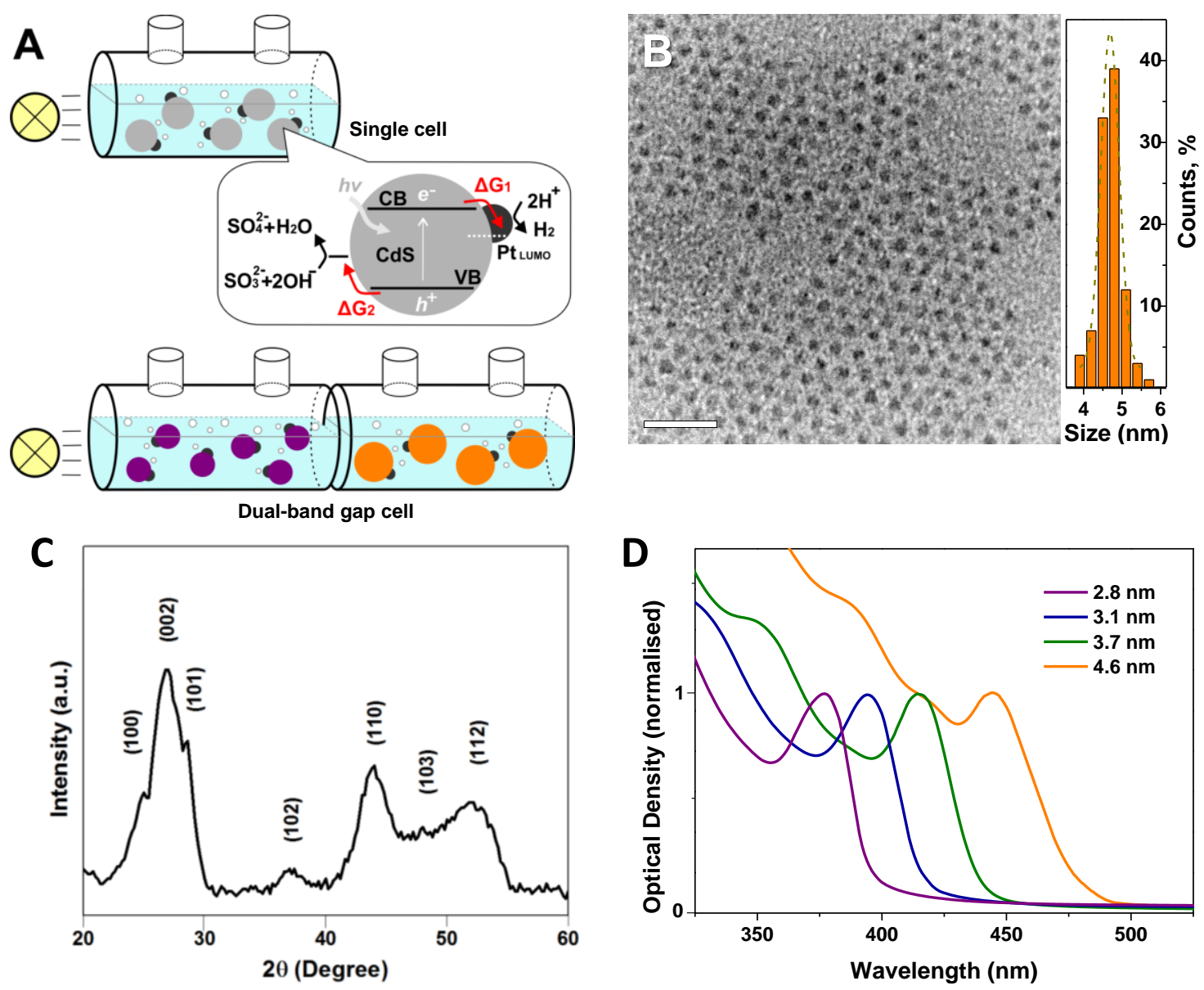

Fig. 1 A) Schematics of the experimental configurations for single-cell and dual-band gap cell photocatalytic experiments. B) TEM image of $4.6 \mathrm{~nm}$ diameter CdS nanospheres along with the size distribution as determined from TEM images. C) XRD pattern of the $4.6 \mathrm{~nm}$ CdS nanospheres along with reflex assignments according to CdS hexagonal wurzite phase (JCPDS card No. 41-1049), D) Extinction spectra of the size-controlled Pt-decorated CdS nanospheres demonstrating strong quantum confinement and band gap widening with decreasing size.

\section{Experimental}

Figure 1a illustrates the setup for photocatalytic experiments. A colloidal aqueous suspension of the Pt-decorated SNCs (pH 9, optical density 1.5 at first excitonic transition) was illuminated by the filtered (350-480 $\mathrm{nm}$ band pass, $50 \mathrm{~mW} / \mathrm{cm}^{2}$ at the sample, see supporting information for illumination spectra) output of a Xe lamp under an Ar atmosphere in a gas-tight cuvette in presence of sodium sulphite as hole scavenger and triethanolamine as stabiliser. ${ }^{14}$ The photogenerated electron transfers to the Pt clusters and reduces protons to generate hydrogen, while the 
photogenerated hole oxidises the sacrificial hole scavenger sodium sulphite. Aliquots of the gas atmosphere were analysed in a gas chromatograph (Bruker 430-GC Gas Chromatograph configured with a TCD detector) for their hydrogen content after well-defined periods of illumination. This allows precise determinations of both internal and external quantum efficiencies. The external and internal quantum efficiency for hydrogen generation are determined as the ratio of $2 x$ number of hydrogen molecules evolved per number of incident and absorbed photons, respectively. The incident power is measured with a thermal power sensor (Thorlabs S302C). The spectrum of the incident light was measured using an Ocean optics USB4000 spectrometer. Convoluting the absorption spectrum of the SNCs with excitation spectrum and taking into account the illumination power allows precise determination of the absorbed photons. Similarly, overall illumination power in combination with the excitation spectrum allows determining the number of incident photons as function of wavelength. The irradiation area was $1.0 \mathrm{~cm}^{2}$ and the irradiation power was $50 \mathrm{~mW}$. The single-cell setup was used to study the size dependence of hydrogen generation efficiency which informed the choice of nanocrystal sizes for the dual-band gap cell experiments. For the dual-band gap cell experiments two cuvettes were placed behind each other; the front one containing $2.8 \mathrm{~nm}$ diameter SNCs (i.e. large band gap) and the rear one $4.6 \mathrm{~nm}$ diameter SNCs (small band gap). The optical density (OD) at the first excitonic transition was kept constant at 1.5 in each cuvette by adjusting the concentration of the SNCs in suspension. Nanospheres of CdS, a widely studied photocatalyst, ${ }^{15-18}$ of four different controlled diameters (2.8/3.1/3.7/4.6 nm as determined from peak position of the first excitonic transition) were synthesised and decorated with sub-nm Pt clusters according to reported procedures. ${ }^{3,19}$ Figure $1 \mathrm{~b}$ displays a transmission electron microscopy (TEM) image of the largest SNCs prepared with a narrow size distribution centred at around $4.7 \mathrm{~nm}$. Corresponding data for the other sizes are available in the supporting information Fig. S1. Figure $1 \mathrm{c}$ displays XRD data for $4.6 \mathrm{~nm}$ CdS nanospheres revealing a hexagonal wurtzite phase of the SNCs. It is noted that the small size of the SNCs leads to a significant broadening of the XRD reflexes. The photodeposition employed here results in sub-nm Pt cluster decoration of the SNCs. At this size the Pt clusters are in the non-scalable size regime, i.e. their optical and electronic properties are discrete and depend on the size of the clusters. In particular, their electronic states can be considered molecule-like in that they exhibit highest occupied molecular and lowest unoccupied molecular orbitals (HOMO and LUMO), respectively. This was further confirmed by the Pt core level binding energies in XPS (see supporting information) which are larger than for metallic Pt. XPS also revealed the bonding of Pt to $\mathrm{Cd}$ in the SNCs. The Pt decoration was kept constant at $\sim 16 \mathrm{wt} \%$ for each sample as verified by inductively coupled plasma-optical emission spectrometry (ICP-OES, see supporting information). Figure $1 \mathrm{~d}$ displays the extinction spectra of the four Pt-decorated samples. A clear blue-shift of the extinction onset and the peak position of the first excitonic transition can be observed with decreasing SNC diameter indicating strong quantum confinement and widening of the band gap in the SNCs. It should be noted that the Pt co-catalyst does not contribute significantly to the total absorption.

\section{Results and discussion}

First, we investigated the relationship between SNC diameter and internal quantum efficiency for hydrogen generation. Fig. 2a displays the internal quantum efficiency (i.e. $2 x$ number of $\mathrm{H}_{2}$ molecules evolved/number of absorbed photons) as a function of SNC diameter. Clearly, the internal quantum efficiency increases with decreasing SNC diameter from $11.4 \%(4.6 \mathrm{~nm})$, via $13.3 \%(3.7 \mathrm{~nm})$ and $15.0 \%(3.1 \mathrm{~nm})$, to $17.3 \%(2.8 \mathrm{~nm})$. As will be discussed in detail in the following we attribute this behaviour to an increase in the energy 
level off-set between SNC and the co-catalyst and hole-scavenger, respectively, which increases the driving forces for electron transfer and hole scavenging ? $\mathrm{G}_{1}{ }$ ? $\mathrm{G}_{2}$ respectively.

Figure $2 \mathrm{~b}$ displays the hydrogen evolution as a function of time for the colloidal dual-band gap cell geometry. It can be clearly seen that employing $2.8 \mathrm{~nm}$ SNCs in the front and $4.6 \mathrm{~nm}$ SNCs in the rear cuvette results in higher overall hydrogen generation than employing the same size nanocrystals in both cuvettes. The external quantum efficiency in this case increases from $9.4 \%$ ( $2.8 \mathrm{~nm}$ in both front and rear cuvette; $2.8 \mathrm{~nm}+2.8 \mathrm{~nm})$ via $11.3 \%(4.6 \mathrm{~nm}+4.6 \mathrm{~nm})$ to $14.7 \%(2.8 \mathrm{~nm}+4.6 \mathrm{~nm})$. It is noted, that in the two setups with same size SNCs in both cuvettes $4.6 \mathrm{~nm}$ SNCs absorb more
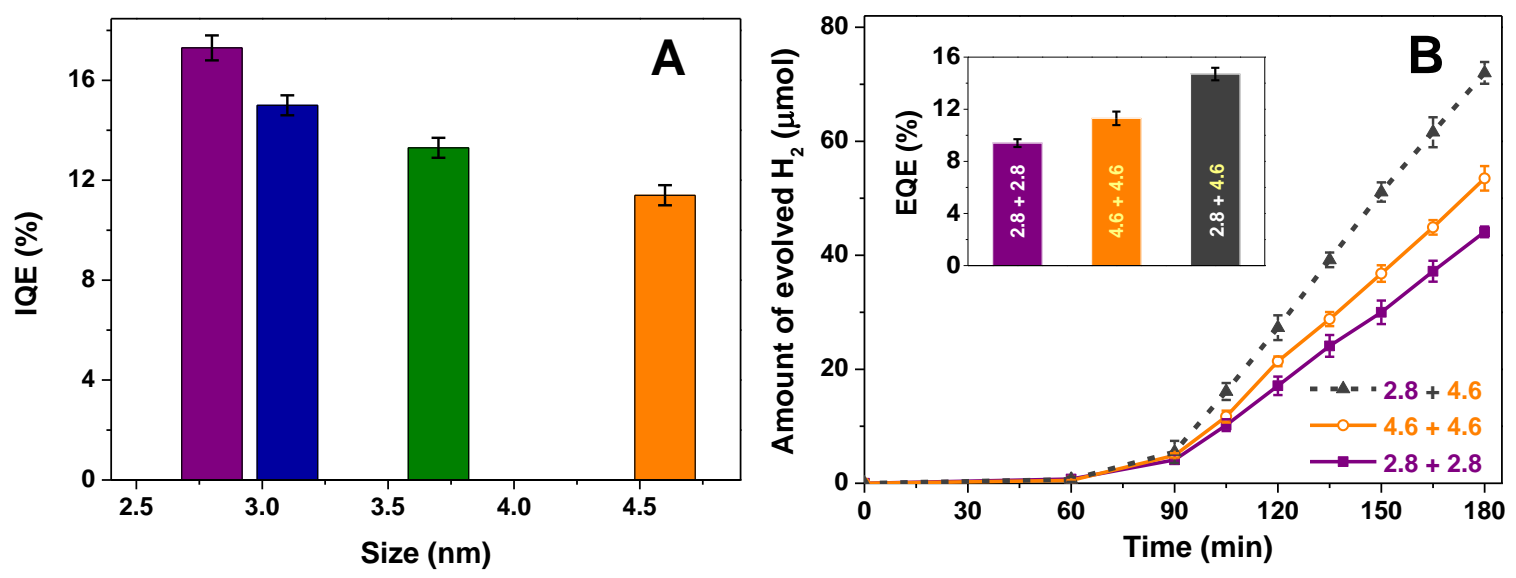

Fig. 2 A) Internal quantum efficiency for photocatalytic hydrogen generation as function of SNC diameter in single-cell geometries. B) Hydrogen evolution as a function of time for dual-band gap cells with $2.8 \mathrm{~nm} \mathrm{SNCs}$ only (purple solid squares), $4.6 \mathrm{~nm}$ SNCs only (orange open circles), and $2.8 \mathrm{~nm}+4.6 \mathrm{~nm}$ SNCs (black solid triangles). Inset: External quantum efficiencies for photocatalytic hydrogen evolution in the same dual-band gap geometries. (In all measurements the OD of the SNC suspensions were kept at 1.5 in each cell at the first excitonic absorption peak resulting in $26-53 \mathrm{mg} / \mathrm{L}$ of Pt-decorated $\mathrm{CdS}$ for the four sizes. Samples were excited with a power of $\sim 50 \mathrm{~mW} / \mathrm{cm} 2$ and spectral range between $350-480 \mathrm{~nm}$. SNC suspension contained $0.02 \mathrm{M}$ sodium sulphite as hole scavenger and $0.05 \mathrm{M}$ triethanolamine as stabiliser with at a $\mathrm{pH}$ of 9).

photons than the $2.8 \mathrm{~nm}$ SNCs, which in the present case overcompensates the lower IQE of the 4.6 $\mathrm{nm}$ particles leading to a higher EQE. During the noticeable induction period the cysteine ligands being present post-synthesis are being replaced by triethanolamine which then acts as a stabiliser in the hydrogen evolution period. ${ }^{14}$

Table 1 reports alternative measures of the photocatalytic activity of the differently sized SNCS namely the formation rate (i.e. amount of hydrogen per hour and gram of $\mathrm{CdS}$ and $\mathrm{Pt}$ ) and turn-overfrequency (TOFs) per Pt atom. The latter have been estimated using the elemental composition of the Pt decorated SNCs from ICP-OES measurements. We note that this number represents a lower limit for the TOFs of each catalytically active site (i.e. Pt cluster). As can be seen both the formation rate and the TOF normalised to the number of Pt atoms increase significantly with decreasing particle size. This demonstrates that the smaller diameter SNCs not only display larger internal quantum efficiencies but also represent a more efficient use of the semiconductor and co-catalyst materials.

Due to their larger band gap, the smaller SNCs absorb a smaller portion of illumination light but convert it to hydrogen with larger efficiency as compared to the larger SNCs. This enables the 
colloidal dual-band gap cell introduced here, where similar to a tandem solar cell cell, ${ }^{20,21}$ the larger SNCs in the rear cuvette absorb the photons that are not used by the larger band gap material in the front. While this reduces the internal quantum efficiency of the dual-band gap cell compared to the smallest SNCs, it increases the external quantum efficiency which is a measure for the efficiency with which the incident photons are used. This is also reflected in the corresponding formation rates of 45.5/77.6/81.9 $\mathrm{mmol} \mathrm{H} / \mathrm{g} / \mathrm{h}$ for $4.6 \mathrm{~nm}+4.6 \mathrm{~nm}, 2.8 \mathrm{~nm}+2.8 \mathrm{~nm}$, and $2.8 \mathrm{~nm}+4.6 \mathrm{~nm}$ dual-band gap cells, respectively. We note that the internal quantum efficiency in the rear cuvette is not affected by the presence of the front cuvette for differently sized SNCs, i.e. it is identical to the single cell measurements and there is no dependence of the internal quantum efficiency on photon energy for a given size of SNCs. For same size SNCs in both cuvettes most of the $\mathrm{H}_{2}$ is generated in the front cuvette with the same internal quantum efficiency as the single cell experiment.

Table 1 Internal quantum efficiency, formation rate, and turn-over-frequency per Pt atom for the four differently sized, Pt decorated CdS nanosphere samples.

\begin{tabular}{llll}
\hline $\begin{array}{l}\text { SNC } \\
\text { diameter } \\
\text { /nm }\end{array}$ & IQE, \% & $\begin{array}{l}\mathrm{H}_{2,} \\
\mathrm{mmol} / \mathrm{g}(\text { catalyst)/h }\end{array}$ & $\begin{array}{l}\mathrm{TOF}, \\
\mathrm{H}_{2} / \mathrm{Pt} / \mathrm{s}\end{array}$ \\
\hline 2.8 & 17.3 & 155 & 0.045 \\
3.1 & 15.0 & 136 & 0.038 \\
3.7 & 13.3 & 111 & 0.030 \\
4.6 & 11.4 & 85.9 & 0.024 \\
\hline
\end{tabular}

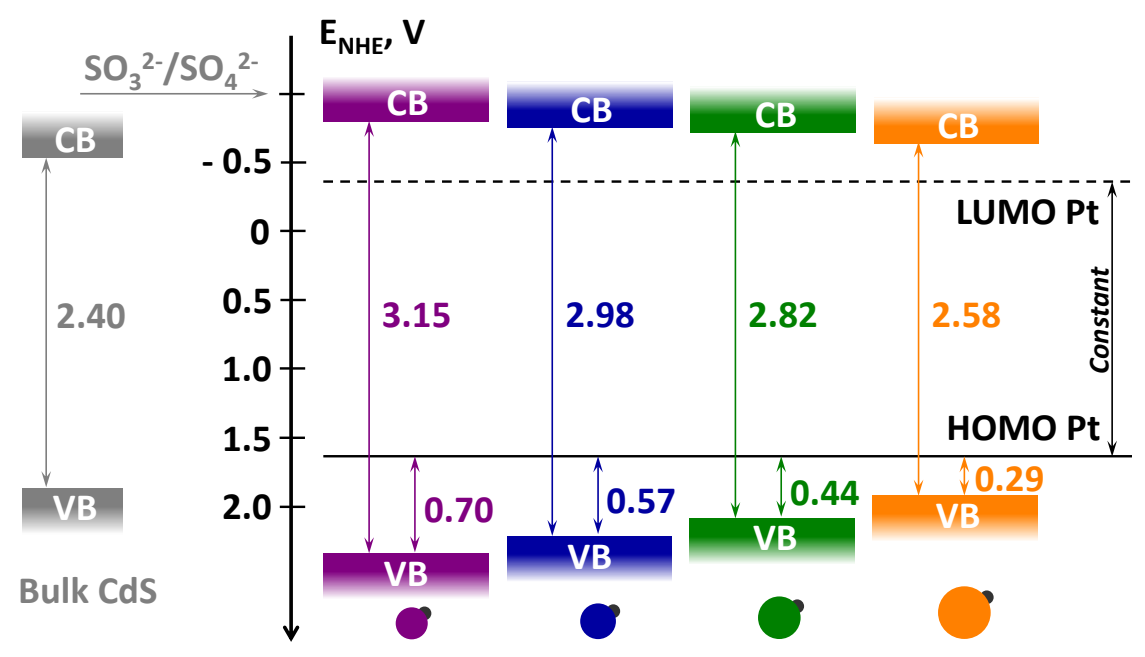

Fig. 3 Band diagram of the conduction and valence band edges of Pt-decorated CdS nanospheres with diameters of 2.8 (purple)/3.1 (blue)/3.7 (green)/4.6 (orange) nm and Pt HOMO positions as determined from XPS and extinction measurements. The Pt LUMO is as a guide to eye only assuming a constant HOMO-LUMO-gap for the sub-nm Pt clusters across the differently sized SNCs. The valence and conduction band edges for bulk CdS, to which the experimental data have been aligned, ${ }^{24}$ and the redox level of the hole scavenger at $\mathrm{pH} 0$ are taken from references 7,19 . Proton reduction occurs at $\mathrm{OV}$ on the NHE scale. For details see text.

We propose here that the increase in internal quantum efficiency with decreasing size is due to an increased driving force $\mathrm{G}_{1} /$ ? $\mathrm{G}_{2}$ for both the electron transfer to the $\mathrm{Pt}$ and the hole 
scavenging mechanism. The increase in driving forces results directly from a larger energy level off-set between SNC and Pt/hole scavenger due to the larger band gaps in the smaller SNCs. To substantiate this attribution we determined a band diagram (Figure 3 ) based on XPS and optical data. The relative off-sets of the valence band edges of the SNCs in Figure 3 were determined from the leading edge of the XPS spectra of Pt-decorated SNCs and undecorated SNCs, respectively. To correct for charging and quantum confinement effects in the XPS data $^{22}$, we aligned the XPS spectra of the Pt decorated samples at the valence band edge assuming similar energetic positions for the highest occupied molecular orbital of the sub-nm Pt clusters across the differently sized SNCs. The XPS spectra of the undecorated SNCs were then aligned with respect to the Cd $3 d$ core levels of the decorated SNCs of the same diameter. The relative positions of the conduction band edges were then determined by the optical band gap obtained from extrapolation of the absorption onset in the data displayed in Figure $1 \mathrm{~b}$. Finally, the experimental XPS data were aligned to the known CdS bulk band edges $^{23}$ (with respect to the normal hydrogen electrode (NHE) scale; $\mathrm{H} / \mathrm{H}^{+}$redox potential at $\mathrm{OV}$ at $\mathrm{pH}$ ) taking into account the size-dependent band edge shifts due to quantum confinement via $\mathrm{E} E=\hbar^{2} \pi^{2} / 2 \mathrm{me}_{\mathrm{h}} \mathrm{R}^{2} .{ }^{24}$

The resulting band diagram (Figure 3) clearly demonstrates the widening band gap of the SNCs in which both conduction and valence band edges shift away from their bulk values. Most importantly the SNC conduction band edge shows an increased offset with respect to the Pt LUMO. ${ }^{\ddagger}$ The valence band edge, correspondingly, shows an increased offset with respect to the redox potential of the hole scavenger. The determined band edge positions suggest that the driving forces for electron transfer will increase by $90 / 30 / 40 \mathrm{meV}$ when comparing the SNCs of decreasing size. The corresponding driving forces for hole scavenging increase by 150/130/130meV, respectively.

\section{Conclusions}

In conclusion, we demonstrate a colloidal dual-band gap cell for photocatalytic hydrogen generation using size-controlled, Pt-decorated CdS nanospheres and report increases in external quantum efficiency by more than $50 \%$ compared to single-size SNC-cells. The colloidal dual-band gap cell demonstrated here is based on the size-dependent photocatalytic activity of the SNCs where smaller SNCs convert the absorbed light more efficiently into hydrogen. This effect is based on increased driving forces for electron transfer and hole scavenging, respectively, which in turn originates in the larger, quantum confinement-induced band offsets between SNC and co-catalyst/hole scavenger, respectively. Our results represent a proof-ofprinciple for future colloidal tandem cells and will be applicable to a range of photocatalytic reactions including $\mathrm{CO}_{2}$ reduction for solar fuel generation as well as other material systems.

\section{Acknowledgements}

Financial support by The University of Liverpool (F.J.) is gratefully acknowledged. YG acknowledges financial support from the Royal Society through a University Research Fellowship. GO'D, DH, TJW acknowledge funding through EPSRC PhD studentships.

\section{Notes and references}

\# It should be noted that the LUMO position is only intended as a guide to the eye as we assume a constant HOMO LUMO-gap for the Pt clusters across the differently sized SNCs because experimental data for it are not available. 
1 N. S. Lewis and D. G. Nocera, Proc. Natl. Acad. Sci., 2006, 103, 15729.

2 L. Amirav and A. P. Alivisatos, J. Phys. Chem. Lett., 2010, 1, 1051.

3 M. Berr, A. Vaneski, A. S. Susha, J. Rodríguez-Fernández, M. Döblinger, F. Jäckel, A. L. Rogach and J. Feldmann, Appl. Phys. Lett., 2010, 97, 093108.

4 K. Maeda and K. Domen, J. Phys. Chem. Lett. 2010, 1, 2655.

5 F. E. Osterloh, Chem. Soc. Rev., 2013, 42, 2294.

6 K. P. Acharya, R. S. Khnayzer, T. O'Connor, G. Diederich, M. Kirsanova, A. Klinkova, D. Roth, E. Kinder, M. Imboden and M. Zamkov, Nano Lett., 2011, 11, 2919.

7 M. J. Berr, P. Wagner, S. Fischbach, A. Vaneski, J. Schneider, A. S. Susha, A. L. Rogach, F. Jäckel and J. Feldmann, Appl. Phys. Lett., 2012, 100, 223903.

8 T. Simon, N. Bouchonville, M. J. Berr, A. Vaneski, A. Adrović, D. Volbers, R. Wyrwich, M. Döblinger, A. S. Susha, A. L. Rogach, F. Jäckel, J. K. Stolarczyk and J. Feldmann, Nat. Mater., 2014, 13, 1013.

9 K. Tvrdy, P. A. Frantsuzovc and P. V. Kamat, Proc. Natl. Acad. Sci., 2011, 108, 29.

10 J. Zhao, M.A. Holmes and F. E. Osterloh, ACS Nano, 2013, 7, 4316.

11 K. A. Brown, Q. Song, D. W. Mulder and P. W. King, ACS Nano, 2014, 8, 10790.

12 U. Soni, P. Tripathy and S. Sapra, J. Phys. Chem. Lett., 2014, 5, 1909.

13 S. N. Habisreutinger, L. Schmidt-Mende and J. K. Stolarczyk, Angew. Chem. Int. Ed., 2013, 52, 7372.

14 N. Mntungwa, V. S. Rajasekhar Pullabhotla and N. Revaprasadu, Colloids Surf. B Biointerfaces, 2013, 101, 450.

15 Q. Li, B. Guo, J. Yu, J. Ran, B. Zhang, H. Yan and J. R. Gong, J. Amer. Chem. Soc., 2011, 133, 10878.

16 Z.-Q. Qina and F.-J. Zhang, Appl. Surf. Sci., 2013, 285P, 912.

17 Y. Zhua, Y. Wang, Z. Chen, L. Qin, L. Yang, L. Zhu, P. Tang, T. Gao, Y. Huang, Z. Sha and G. Tang, App. Cat. A, 2015, 498, 159.

18 H. Wang, W. Chen, J. Zhang, C. Huang and L. Mao, Intern. J. Hydr. Ener, 2015, 40, 340.

19 W. W. Yu and X. Peng, Angew. Chem. Int. Ed., 2002, 41, 2368.

20 X. Wang, G. I. Koleilat, J. Tang, H. Liu, I. J. Kramer, R. Debnath, L. Brzozowski, D. A. R. Barkhouse, L. Levina, S. Hoogland and E. H. Sargent, Nat. Photonics, 2011, 5, 480.

21 J. Brillet, J.-H. Yum, M. Cornuz, T. Hisatomi, R. Solarska, J. Augustynski, M. Graetzel and K. Sivula, Nat. Photonics, 2012, 6, 824.

22 M. N. Ghazzal, R. Wojcieszak, G. Raj and E. M. Gaigneaux, Beilstein J. Nanotechnol., 2014, 5, 68.

23 Y. Xu and M. A. A. Schoonen, Amer. Miner., 2000, 85, 543.

V. L. Colvin, A. P. Alivisatos and J. G. Tobin, Phys. Rev. Lett., 1991, 66, 2786. 